\title{
One-Class-at-a-Time Removal Sequence Planning Method for Multiclass Classification Problems
}

\author{
Chieh-Neng Young, Chen-Wen Yen, Yi-Hua Pao, and Mark L. Nagurka
}

\begin{abstract}
Using dynamic programming, this work develops a one-class-at-a-time removal sequence planning method to decompose a multiclass classification problem into a series of two-class problems. Compared with previous decomposition methods, the approach has the following distinct features. First, under the oneclass-at-a-time framework, the approach guarantees the optimality of the decomposition. Second, for a $K$-class problem, the number of binary classifiers required by the method is only $K-1$. Third, to achieve higher classification accuracy, the approach can easily be adapted to form a committee machine. A drawback of the approach is that its computational burden increases rapidly with the number of classes. To resolve this difficulty, a partial decomposition technique is introduced that reduces the computational cost by generating a suboptimal solution. Experimental results demonstrate that the proposed approach consistently outperforms two conventional decomposition methods.
\end{abstract}

Index Terms-Dynamic programming, multiclass classification, pattern recognition.

\section{INTRODUCTION}

A CLASSIFICATION problem deals with objects or events to be classified. Such a problem assumes the existence of a known set of $K$ classes

$$
\boldsymbol{C}=\left\{C_{1} C_{2} \ldots C_{K}\right\}
$$

where $\boldsymbol{C}$ is the set of known classes and the elements $C_{k}$ of $C$ are called classes. A class can be defined as a pair of variables

$$
\text { Pattern }=\left[x, C_{k}\right]
$$

where $\boldsymbol{x}$ is the feature vector that characterizes the property of $C_{k}$. The goal of classification is to find a decision boundary in the feature space in order to recognize the class $C_{k}$ when a feature vector $\boldsymbol{x}$ is present. This mapping can be constructed by a learning-from-example approach where samples with known classes are given. A classifier can then be designed to find the decision boundary in order to infer the class of unknown samples.

A direct approach for multiclass classification problems is to use a single classifier to try to distinguish all classes simultaneously. To adapt neural networks to such problems, an ap-

Manuscript received January 9, 2005; revised October 6, 2005. This work was supported in part by the National Science Council of Republic of China under Grant NSC 87-2212-E-110-007.

C.-N. Young, C.-W. Yen, and Y.-H. Pao are with the Department of Mechanical and Electro-Mechanical Engineering, National Sun Yat-Sen University, Kaohsiung 80424, Taiwan, R.O.C. (e-mail: opt@cdpa.nsysu.edu.tw; vincen@mail.nsysu.edu.tw; Robert_Pao@ asus.com.tw).

M. L. Nagurka is with the Department of Mechanical and Industrial Engineering, Marquette University, Milwaukee, WI 53201-1881 USA (e-mail: mark. nagurka@marquette.edu).

Digital Object Identifier 10.1109/TNN.2006.879768 proach is to assign a binary string to each class as the target output. To enhance its performance, these binary strings can be designed with error-correction [1]-[3], so that errors by a few of the binary numbers can be recovered. Nevertheless, separating many classes at one time is still a very challenging task since the complexity of the decision boundary often increases with the number of classes. In responding to this difficulty, two decomposition methods have been proposed. The basic idea of these methods is to convert a multiclass problem $(K \geq 3)$ into a number of two-class problems $(K=2)$. In this work, the two classes associated with a two-class problem are referred to as the true and the false classes, respectively.

The one-against-all (1-a-a) method (e.g., [4]) converts a $K$-class problem into $K$ two-class problems. In particular, the $i$ th binary classifier used by the $1-\mathrm{a}-\mathrm{a}$ method is designed by choosing $C_{i}$ as the true class, whereas the union of the remaining classes (denoted as $\overline{C_{i}}$ ) is the false class. A sample is assigned to $C_{i}$ when the $i$ th binary classifier has the largest true class output. Despite the fact that the 1-a-a method only needs to solve two-class problems one at a time, the training sets of all these two-class problems still come from the union of the same large number of classes associated with the original multiclass problem. As a result, many of the converted two-class problems can still be very difficult to solve.

By trying to distinguish every set of $C_{i}$ from $C_{j}$ for $i, j=$ $1 \ldots, K$ and $i>j$, the one-against-one (1-a-1) method (e.g., [5]) splits a $K$-class problem into $K(K-1) / 2$ two-class problems. In performing a classification, the 1-a-1 method assigns a sample to the class that has won the largest number of true class votes. Compared with the two-class problems of the 1-a-a method, the two-class problems of the 1-a-1 method are often easier to solve since the decision boundary between $C_{i}$ and $C_{j}$ is expected to be less complex than the decision boundary that separates $C_{i}$ and $\overline{C_{i}}$ (which contains $C_{j}$ as well as all the remaining classes). This observation is supported by several experimental results (e.g., [3], [6]). A tradeoff of this improvement is that the number of classifiers increases from $K$ to $K(K-1) / 2$. Another problem of the 1-a-1 method comes from the ineffective results produced by some of its classifiers. Specifically, a binary classifier trained by samples from $C_{i}$ and $C_{j}$ can produce unreliable classification results if it is used to determine the membership of $C_{k}$ samples for $k \neq j$ and $k \neq i$. This "ineffective decision" problem will be addressed again in Section III.

A goal of this work is to develop an alternative decomposition method that requires fewer classifiers than the 1-a-a method and achieves higher classification accuracy than the 1-a-1 method. The paper is organized as follows. The basic idea of the proposed approach is presented in Section II. To reduce the computational cost and improve the classification accuracy, 


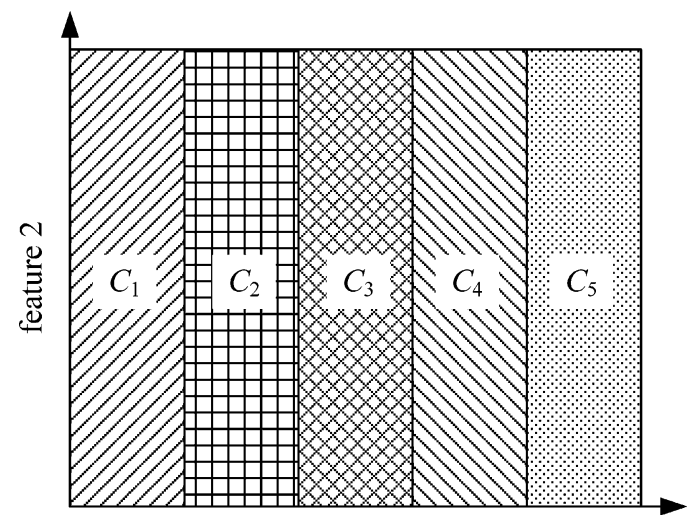

feature 1

Fig. 1. Artificial five-class problem.

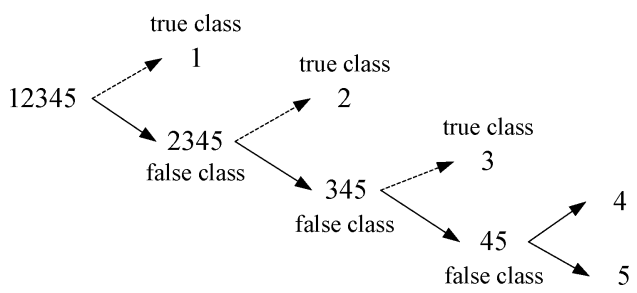

Fig. 2. One-class-at-a-time removal sequence for a five-class problem.

two variations of the approach are introduced in Section III. Section IV presents experimental results that demonstrate the efficiency and accuracy of the methods, and conclusions are given in Section V.

\section{ONE-CLASS-AT-A-TIME APPROACH}

To perform the decomposition, in the proposed approach, a binary classifier is first designed for $C_{i}$ and $\overline{C_{i}}$ for every $C_{i}$. Next, for every $\overline{C_{i}}$, a binary classifier is developed to classify $C_{j}$ and $\overline{\boldsymbol{C}_{i j}}$ for every $j \neq i$. Note that $\overline{\boldsymbol{C}_{i j}}$ represents a class obtained by removing $C_{i}$ and $C_{j}$ from $\boldsymbol{C}$. This one-class-at-atime removal procedure is continued until all classes have been classified. This procedure requires only $K-1$ binary classifiers.

The critical issue of this one-class-at-a-time approach is the planning of the removal sequence. It is very likely that a cleverly arranged sequence can simplify the classification problem. To illustrate this possibility, an artificial problem of separating five classes based on two features, as shown in Fig. 1, is considered. To tackle this problem, it is assumed that $C_{1}, C_{2}, C_{3}$, and $C_{4}$ can be removed one-at-a-time from the training set, as illustrated in Fig. 2. For simplicity, this work uses a sequence of $12345 \rightarrow 2345 \rightarrow 345 \rightarrow 45$ to represent the removal sequence of Fig. 2. From Fig. 1, it is easy to see that the four two-class problems associated with this removal sequence are all linearly separable and thus easy to solve. In contrast, a nonlinear decision boundary is required in trying to perform an operation of $12345 \rightarrow 1245$ to separate $C_{3}$ and $\overline{C_{3}}$.

Searching an optimal removal sequence for this problem can be formulated as a multistage decision-making problem (e.g., [7]). In particular, a $K$-class problem can be decomposed into a decision-making problem of $K-1$ stages. Fig. 3 depicts such a multistage decision-making problem for a five-class

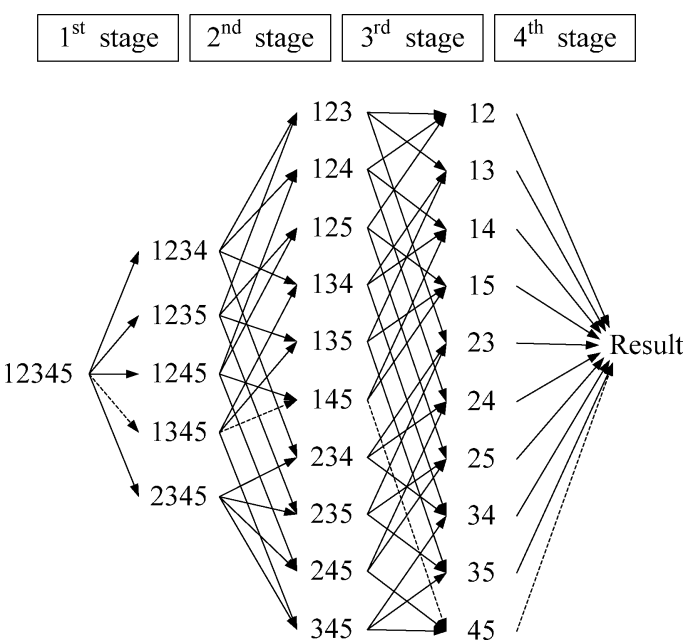

Fig. 3. Tree structure containing all possible one-class-at-a-time solutions for a five-class problem.

problem. Essentially, each path that connects the starting and ending nodes of Fig. 3 represents a possible one-class-at-a-time solution for the given multiclass problem. In addition, the tree structure (also called version space in machine learning literature) of Fig. 3 contains all such possible solutions. For each stage, the decision that must be made is which class to be removed from the remaining training set so that the cost function can be minimized. Since the goal is to minimize the classification error, this work specifies the cost function as the number of misclassified samples.

Based on the principle of optimality, dynamic programming (DP) can be used to find the global optimal solution for the multistage decision-making problem. For the multiclass classification problem under consideration, the principle of optimality yields the following recurrence relation:

$$
\begin{aligned}
J^{*}\left(\boldsymbol{C}_{p}^{M}\right)=\min _{q}\left[J\left(\boldsymbol{C}_{p}^{M} \rightarrow\left(\boldsymbol{C}_{p}^{M}-\boldsymbol{C}_{p q}^{M}\right)\right)\right. & \\
& \left.+J^{*}\left(\boldsymbol{C}_{p}^{M}-\boldsymbol{C}_{p q}^{M}\right)\right]
\end{aligned}
$$

for $M=3,4, \ldots K$. Here, the asterisk indicates that the function has been optimized and thus has the optimal value. In addition, $\boldsymbol{C}_{p}^{M}$ represents the $p$ th combination of $M$ classes from the $K$ classes of $\boldsymbol{C}, C_{p q}^{M}$ is the $q$ th member class of $\boldsymbol{C}_{p}^{M}$, and $J^{*}\left(\boldsymbol{C}_{p}^{M}\right)$ and $J^{*}\left(\boldsymbol{C}_{p}^{M}-C_{p q}^{M}\right)$ are the costs associated with the optimal one-class-at-a-time removal sequences for $\boldsymbol{C}_{p}^{M}$ and $\boldsymbol{C}_{p}^{M}-C_{p q}^{M}$, respectively. Also, the term $J\left(\boldsymbol{C}_{p}^{M} \rightarrow\left(\boldsymbol{C}_{p}^{M}-C_{p q}^{M}\right)\right)$ is the cost associated with the operation of classifying $\boldsymbol{C}_{p}^{M}$ into $C_{p}^{M}-C_{p q}^{M}$ and $C_{p q}^{M}$. Since the last decision for the proposed one-class-at-a-time approach is to remove a class from a three-class problem, the solution process is initiated by setting $M$ equal to 3 . As a result, (1) can be written as

$$
J^{*}\left(C_{p}^{3}\right)=\min _{q}\left[J\left(C_{p}^{3} \rightarrow\left(C_{p}^{3}-C_{p q}^{3}\right)\right)+J^{*}\left(C_{p}^{3}-C_{p q}^{3}\right)\right] .
$$

After removing $C_{p q}^{3}$ from $C_{p}^{3}, C_{p}^{3}-C_{p q}^{3}$ contains only two classes. As a result, $J^{*}\left(C_{p}^{3}-C_{p q}^{3}\right)$ can be determined by sepa- 
rating every possible set of $C_{i}$ and $C_{j}$ for $i, j=1 \ldots, K$ and $i>j$. Note that the 1-a-1 method also solves the same classification problems.

By applying the recurrence relation recursively, the solution procedure is continued until $M=K$. When $M=K$, (1) can be written as

$$
\begin{aligned}
J^{*}\left(\boldsymbol{C}_{p}^{K}\right)=\min _{q}\left[J \left(\boldsymbol { C } _ { p } ^ { K } \rightarrow \left(\boldsymbol{C}_{p}^{K}\right.\right.\right. & \left.\left.-\boldsymbol{C}_{p q}^{K}\right)\right) \\
& \left.+J^{*}\left(\boldsymbol{C}_{p}^{K}-\boldsymbol{C}_{p q}^{K}\right)\right] .
\end{aligned}
$$

Note that there is only one possible $C_{p}^{K}$, which is the union of all classes, that is, $\boldsymbol{C}$. Therefore, (3) can be rewritten as

$$
J^{*}(\boldsymbol{C})=\min _{q}\left[J\left(\boldsymbol{C} \rightarrow \overline{\boldsymbol{C}}_{q}\right)+J^{*}\left(\overline{\boldsymbol{C}}_{q}\right)\right]
$$

with $q=1, \ldots, K$. Note that the binary classifiers associated with $J\left(\boldsymbol{C} \rightarrow \overline{\boldsymbol{C}_{q}}\right)$ are identical to those employed by the 1-a-a method.

The results demonstrate that the binary classifiers developed by the conventional 1-a-a and 1-a-1 methods are only a subset of the classifiers used by the proposed approach. For example, for the five-class problem of Fig. 3, the classifiers employed by the 1-a-1 correspond to the rightmost arrows, whereas the leftmost arrows represent the binary classifiers used by the 1-a-a method.

A distinct advantage of DP, compared with other optimization strategies such as a genetic algorithm, is that it guarantees a global optimal solution. Therefore, if the one-class-at-a-time requirement is relaxed, DP can find better solutions. The tradeoff is that the number of possible solutions increases dramatically with the number of classes and the problem then may become computationally too intensive to solve. The proposed approach represents a compromise between computational cost and classification accuracy.

\section{Two VARIATIONS OF THE Proposed APPROACH}

\section{A. A Suboptimal Version of the Proposed Approach}

By searching the entire solution space systematically, DP guarantees global optimality for the one-class-at-a-time removal sequence. However, to perform such a search, it can be shown that the number of converted two-class problems is $\sum_{i=0}^{K-3}\left(\begin{array}{c}K \\ K-i\end{array}\right)(K-i)+\left(\begin{array}{c}K \\ 2\end{array}\right)$. This number increases rapidly with the number of classes $K$ hindering the application of the proposed approach to problems with a large number of classes.

To address this "curse of dimensionality" difficulty, this work proposes a suboptimal approach by applying the one-class-at-atime decomposition only to a subset of $\boldsymbol{C}$ and uses the conventional 1-a-1 method to classify the remaining classes. The specific steps of this suboptimal approach are as follows.

1) Based upon the available computing power, determine the number of classes that is computationally feasible for the proposed approach. Let this number be $M$.

2) Apply the 1-a-1 method to the given $K$-class problem.

3) For every $M$-class subset of the given $K$ classes, determine the number of internal classification errors, which is the number of training samples that are incorrectly assigned to a class that belongs to the same $M$-class subset.

4) Find the $M$-class subset that has the largest number of internal classification errors. Denote this set of $M$ classes as $\widetilde{\boldsymbol{C}}^{M}$. The union of the remaining classes is, therefore, $C-\widetilde{C}^{M}$.

5) Apply the proposed one-class-at-a-time approach to decompose $\widetilde{\boldsymbol{C}}^{M}$.

6) To classify a sample, the 1-a-1 method is used first. The classification result is accepted provided that the sample is assigned to one of the classes of $\boldsymbol{C}-\widetilde{\boldsymbol{C}}^{M}$. Otherwise, the sample is classified again by using the one-class-at-a-time removal sequence developed in step 5).

This technique does not guarantee a true optimal solution since it disregards the errors of assigning $\widetilde{\boldsymbol{C}}^{M}$ samples to $\boldsymbol{C}_{-}$ $\widetilde{\boldsymbol{C}}^{M}$ and the errors of misclassifying $\boldsymbol{C}-\widetilde{\boldsymbol{C}}^{M}$ samples to $\widetilde{\boldsymbol{C}}^{M}$. However, by replacing the 1-a-1 method with the proposed decomposition approach for classifying $\widetilde{\boldsymbol{C}}^{M}$ (which has the largest internal classification error among all the $M$-class subset), it is expected that the number of internal classification errors of $\widetilde{\boldsymbol{C}}^{M}$ can be reduced, thus effectively improving the overall classification accuracy.

\section{B. Building a Committee Machine}

Based on the fusion of multiple classifiers, it has been shown that a committee machine can provide higher classification accuracy than an individual classifier (e.g., [8] and [9]). As an example, the 1-a-1 method is essentially a committee machine with $K(K-1) / 2$ binary classifier members. Thereafter, the symbol $B(i, j)$ is used to represent such a binary classifier that is trained to classify $C_{i}$ and $C_{j}$.

As described in Section I, one weakness of the 1-a-1 method is the ineffective decision problem, which occurs when trying to use a classifier $B(i, j)$ to classify samples that do not belong to $C_{i}$ or $C_{j}$. The goal of this subsection is to introduce a new committee machine to resolve the ineffective decision problem by using the one-class-at-at-a-time technique.

Let $S_{i j}$ represent the one-class-at-a-time removal sequence that has $B(i, j)$ as its final classifier. The basic idea of this new committee machine is to use the first $K-2$ binary classifiers of $S_{i j}$ to "filter out" samples that do not belong to $C_{i}$ or $C_{j}$. By requiring $C_{i}$ and $C_{j}$ to be the last two classes to be processed, the removal sequence $S_{i j}$ can be determined by rewriting the recurrence relation of (1) as

$$
\begin{array}{r}
J^{*}\left(\boldsymbol{C}_{p}^{M(i, j)}\right)=\min _{q}\left[J\left(\boldsymbol{C}_{p}^{M(i, j)} \rightarrow\left(\boldsymbol{C}_{p}^{M(i, j)}-\boldsymbol{C}_{p q}^{M(i, j)}\right)\right)\right. \\
\left.+J^{*}\left(\boldsymbol{C}_{p}^{M(i, j)}-\boldsymbol{C}_{p q}^{M(i, j)}\right)\right]
\end{array}
$$

for $M=4, \ldots K$. The definition of $C_{p}^{M(i, j)}$ is similar to $\boldsymbol{C}_{p}^{M}$ except that $C_{p}^{M(i, j)}$ must contain $C_{i}$ and $C_{j}$. Similarly, $C_{p}^{M(i, j)}$ is the $q$ th member class of $C_{p}^{M(i, j)}$. Note that $C_{p}^{M(i, j)} \neq C_{i}$ or $C_{j}$ since $C_{i}$ and $C_{j}$ have to be the last two classes to be classified. 
To initiate the recurrence relation of (5) for a particular set of $i$ and $j$, the following results are first set up for $k=1 \ldots, K$, $k \neq j$, and $k \neq i$ :

$$
\begin{array}{r}
J^{*}\left(\left(\boldsymbol{C}_{i} \cup \boldsymbol{C}_{j} \cup \boldsymbol{C}_{k}\right)\right)=J\left(\left(\boldsymbol{C}_{i} \cup \boldsymbol{C}_{j} \cup \boldsymbol{C}_{k}\right) \rightarrow\left(\boldsymbol{C}_{i} \cup \boldsymbol{C}_{j}\right)\right) \\
+J\left(\boldsymbol{C}_{i} \cup \boldsymbol{C}_{j}\right) .
\end{array}
$$

This yields $J^{*}\left(\boldsymbol{C}_{p}^{M(i, j)}-C_{p q}^{M(i, j)}\right)$ for $M=4$. Relation (5) can then be applied recursively until $M=K$.

The proposed committee machine uses the same set of classifiers $B(i, j)$ as the 1 -a-1 method. However, instead of using $B(i, j)$ directly, the committee machine uses $S_{i j}$ to determine the "effectiveness" of $B(i, j)$ 's decision. In particular, the first $K-2$ classifiers of $S_{i j}$ can be viewed as a $\overline{\boldsymbol{C}_{i j}}$ sample filter, which can prevent $B(i, j)$ from processing $\overline{\boldsymbol{C}_{i j}}$ samples. Therefore, in this committee machine, the classifier $B(i, j)$ attends voting only when the sample is not assigned to other classes by $S_{i j}$ before reaching classifier $B(i, j)$. Apparently, the reliability of this voting depends on the efficacy of $S_{i j}$ in filtering $\overline{\boldsymbol{C}_{i j}}$ samples. To evaluate its performance, $S_{i j}$ is used to classify the entire training set and count the samples that actually enter the final classification stage of $S_{i j}$. Among these samples, the ratio of $\overline{\boldsymbol{C}_{i j}}$ samples is computed and denoted as $w_{i j}$. Since this ratio characterizes the likelihood of an "ineffective decision," the weighting coefficient for $B(i, j)$ 's voting is chosen as $1-w_{i j}$. Finally, the membership of a sample is determined by counting the weighted votes from all the binary classifiers of $B(i, j)$.

In performing a classification, the basic version of the proposed approach uses $K-1$ binary classifiers. In contrast, with $K(K-1) / 2$ members, the committee machine version of the proposed approach uses $K(K-1)^{2} / 2$ binary classifiers. As a result, the computational requirement will increase proportionally. This factor should be taken into consideration when implementing the proposed committee machine approach for real-time classification tasks.

\section{EXPERIMENTAL RESULTS}

The first part of this section compares the approach developed in Section II with three conventional methods by using them to solve ten real-world problems obtained from the University of California at Irvine, Irvine, repository of machine learning databases and domain theories [10]. The contents of these ten data sets are summarized in Table I.

In testing these methods, the multilayered perceptron (MLP) is chosen as the base classifier [11]-[13]. The numbers of hidden layers and units are chosen as one and five, respectively. No effort has been made to optimize the structure of the MLP. The initial weights are generated randomly from a uniform distribution between 0-0.1. The MLP error measure is chosen as the conventional mean square error with the error defined as the difference between the desired and actual outputs. In this study, the MLP is trained by adaptive boosting (AdaBoost) [14]. The reason for using AdaBoost is that many empirical studies have shown that it can significantly improve the performance of the neural classifiers and is relatively insensitive to overfitting (e.g., [15]).

In comparing the tested methods, the data set is divided into training, validation and testing subsets with an 8:1:1 ratio. The
TABLE I

SUMMARY OF THE TESTED DATA SETS

\begin{tabular}{lccc}
\hline \hline Dataset & $\begin{array}{c}\text { Number of } \\
\text { Samples }\end{array}$ & $\begin{array}{c}\text { Number of } \\
\text { Classes }\end{array}$ & $\begin{array}{c}\text { Number of } \\
\text { Features }\end{array}$ \\
\hline Iris & 150 & 3 & 4 \\
Balance-scale & 625 & 3 & 4 \\
Lymphography & 148 & 4 & 18 \\
Hypothyroid & 3372 & 4 & 28 \\
Vehicle & 846 & 4 & 18 \\
Car-evaluation & 1728 & 4 & 6 \\
Satimage & 6435 & 6 & 36 \\
Glass & 214 & 6 & 9 \\
Segmentation & 2310 & 7 & 18 \\
Yeast & 1484 & 10 & 8 \\
Pendigits & 10992 & 10 & 16 \\
Vowel & 990 & 11 & 10 \\
Krk & 28056 & 18 & 6 \\
\hline \hline
\end{tabular}

TABLE II

ClassificATION ACCURACY AND COMPUTATIONAL COST OF THE SINGLE ClASSIFIER

\begin{tabular}{lcr}
\hline \hline Dataset & $\begin{array}{c}\text { Classification } \\
\text { Accuracy }\end{array}$ & $\begin{array}{c}\text { Computing Time } \\
(\mathrm{sec})\end{array}$ \\
\hline Iris & $96.67 \pm 4.43$ & 2.2 \\
Balance-scale & $90.64 \pm 3.47$ & 13.5 \\
Lymphography & $82.50 \pm 8.94$ & 3.5 \\
Hypothyroid & $96.50 \pm 1.13$ & 188.8 \\
Vehicle & $80.52 \pm 4.10$ & 40.5 \\
Car-evaluation & $92.30 \pm 1.77$ & 54.9 \\
Satimage & $86.21 \pm 1.29$ & 232.2 \\
Glass & $64.33 \pm 10.05$ & 6.4 \\
Segmentation & $93.13 \pm 1.73$ & 117.0 \\
Yeast & $56.22 \pm 3.57$ & 65.2 \\
Pendigits & $94.66 \pm 0.70$ & 513.9 \\
Vowel & $73.18 \pm 3.97$ & 128.7 \\
Krk & $39.61 \pm 0.93$ & 2462.8 \\
\hline \hline
\end{tabular}

training subset is used to adjust the connection weights of the MLP. The validation subset is used by the early-stop technique to avoid overfitting. The testing subset is used to characterize the generalization accuracy of the MLP. For the sake of reliability, the training process is repeated 100 times by using randomly partitioned training, validation, and testing subsets. This paper reports the average of the testing subset classification accuracy.

The experimental studies were performed using an AMD XP $1700+$ based PC. To set up the basis for comparisons, the problems were first solved using a single MLP. Table II presents the means of the classification accuracy and the computing times averaged from 100 trials for each data set. Here, the classification accuracy is defined as the percentage of the correctly classified testing samples. Table III summarizes the means and the standard deviations of the classification accuracy associated with the three decomposition methods. By comparing these results with the results of the single classifier of Table II, it can be seen that the 1-a-a method achieves higher accuracy in six of the ten tested problems and the 1-a-1 method gives a smaller classification error in seven of the ten problems. In contrast, the proposed approach outperforms the single classifier method in all of the tested problems. In addition, the proposed approach has the smallest classification error in all but the last tested problem.

To compare the computational cost, Table IV summarizes the computing time ratio of the three decomposition methods. Here, the computing time ratio is defined as the ratio of computing 
TABLE III

SUMMARY OF ClassificATION ACCURACY FOR THE TESTED ClASSIFICATION PROBLEM $(K=10)$

\begin{tabular}{lccc}
\hline \hline \multirow{2}{*}{ Dataset } & \multicolumn{3}{c}{ Tested Methods } \\
\cline { 2 - 4 } & 1 -a-a & $1-\mathrm{a}-1$ & 1 -at-a-time \\
Iris & $95.81 \pm 5.23$ & $95.53 \pm 5.59$ & $96.67 \pm 4.43$ \\
Balance-scale & $94.42 \pm 3.06$ & $95.21 \pm 2.98$ & $95.89 \pm 2.76$ \\
Lymphography & $83.18 \pm 8.71$ & $82.54 \pm 8.82$ & $83.21 \pm 9.05$ \\
Hypothyroid & $94.93 \pm 1.01$ & $96.13 \pm 1.39$ & $96.88 \pm 0.81$ \\
Vehicle & $81.02 \pm 3.86$ & $81.93 \pm 3.71$ & $82.43 \pm 4.14$ \\
Car-evaluation & $97.82 \pm 1.11$ & $97.62 \pm 1.36$ & $98.05 \pm 1.06$ \\
Satimage & $89.85 \pm 1.27$ & $90.81 \pm 1.11$ & $90.95 \pm 1.17$ \\
Glass & $45.64 \pm 12.29$ & $61.33 \pm 10.04$ & $66.00 \pm 9.41$ \\
Segmentation & $96.50 \pm 1.13$ & $96.39 \pm 1.33$ & $97.01 \pm 1.14$ \\
Yeast & $50.86 \pm 1.42$ & $57.41 \pm 2.95$ & $56.38 \pm 5.78$ \\
\hline \hline
\end{tabular}

TABLE IV

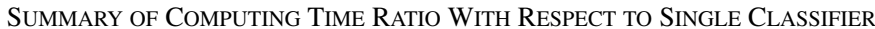
APPROACH $(K=10)$

\begin{tabular}{lccc}
\hline \hline \multirow{2}{*}{ Dataset } & \multicolumn{3}{c}{ Tested Methods } \\
\cline { 2 - 4 } & $1-\mathrm{a}-\mathrm{a}$ & 1 -a-1 & 1-at-a-time \\
\hline Iris & 1.5 & 2.9 & 4.3 \\
Balance-scale & 5.2 & 3.6 & 8.7 \\
Lymphography & 3.1 & 6.6 & 16.2 \\
Hypothyroid & 2.1 & 2.6 & 10.7 \\
Vehicle & 2.3 & 3.6 & 14.3 \\
Car-evaluation & 2.9 & 7.3 & 24.6 \\
Satimage & 6.0 & 21.0 & 197.0 \\
Glass & 2.1 & 2.7 & 50.1 \\
Segmentation data & 3.0 & 7.3 & 210.5 \\
Yeast & 3.7 & 4.0 & 1180.9 \\
\hline \hline
\end{tabular}

time of the tested decomposition method to the computing time of the single MLP. As expected, among the four tested methods, the proposed approach is computationally least efficient. In addition, as shown in Table IV, the computational cost of the proposed approach increases rapidly with the dimension of the classification problem. For example, for the ten-class yeast problem, the computing time of the proposed approach is three orders of magnitude larger than that of the single classifier. In contrast, computationally, the 1-a-a and 1-a-1 methods are only about four times slower than the single classifier.

In responding to this difficulty, the partial decomposition technique is tested on the last four problems of Table I for which the number of classes is ten or greater. In applying the technique, the number of classes to be decomposed $M$ is chosen as 3, 4, 5, and 6. In addition, since the basic version of the proposed approach is less accurate than the 1-a-1 method in dealing with the ten-class yeast problem, the proposed committee machine method is employed in this part of the experiments. The resulting classification accuracies and computing time ratios are summarized in Tables V and VI, respectively.

As shown in Table $\mathrm{V}$, even with partial decomposition, the proposed suboptimal one-class-at-a-time approach outperforms the 1-a-1 method in all four problems. As $M$ increases, the classification accuracy improves. Also, as shown in Table VI, the computational cost also increases with $M$. However, with the partial decomposition, this cost has been reduced significantly. For example, for the ten-class yeast problem, the partial decomposition reduces the computing time ratio from 1180.9 to 36.15 (for $M=6$ or better), demonstrating the effectiveness of the partial decomposition.
TABLE V

SUMmaRy OF ClASSIFICATION ACCURACY FOR THE TESTED CLASSIFICATION PROBLEM $(K=10)$

\begin{tabular}{lccccc}
\hline \hline & \multicolumn{5}{c}{ Method } \\
\cline { 2 - 6 } Dataset & \multirow{2}{*}{$1-\mathrm{a}-1$} & \multicolumn{2}{c}{ One-class-at-a-time } & Committee Machine \\
\cline { 2 - 6 } & & $M=3$ & $M=4$ & $M=5$ & $M=6$ \\
\hline Yeast & $58.09 \pm 3.85$ & $58.10 \pm 3.82$ & $58.24 \pm 3.86$ & $58.42 \pm 3.69$ & $58.58 \pm 3.72$ \\
Pendigits & $98.55 \pm 0.39$ & $98.57 \pm 0.38$ & $98.78 \pm 0.34$ & $98.95 \pm 0.30$ & $99.07 \pm 0.29$ \\
Vowel & $84.74 \pm 4.57$ & $85.08 \pm 4.60$ & $87.19 \pm 4.17$ & $88.59 \pm 3.74$ & $90.23 \pm 3.43$ \\
Krk & $50.50 \pm 0.77$ & $50.36 \pm 0.76$ & $50.70 \pm 0.75$ & $50.84 \pm 0.79$ & $51.06 \pm 0.80$ \\
\hline \hline
\end{tabular}

TABLE VI

Summary of Computing Time Ratio With ResPeCt to Single Classifier APPROACH $(K=10)$

\begin{tabular}{lccccc}
\hline \hline & \multicolumn{5}{c}{ Method } \\
\cline { 2 - 6 } Dataset & \multirow{2}{*}{1 -a-1 } & \multicolumn{3}{c}{ One-class-at-a-time } & Committee Machine \\
\cline { 2 - 6 } & & $M=3$ & $M=4$ & $M=5$ & $M=6$ \\
\hline Yeast & 2.67 & 3.43 & 7.45 & 16.60 & 36.15 \\
Pendigits & 2.44 & 3.67 & 6.19 & 14.73 & 37.91 \\
Vowel & 3.30 & 3.65 & 6.14 & 12.95 & 38.14 \\
Krk & 2.88 & 3.20 & 4.32 & 6.98 & 13.56 \\
\hline \hline
\end{tabular}

\section{CONCLUSION}

This paper proposes a one-class-at-a-time method to decompose a multiclass problem into a number of two-class problems. In particular, the basic version of the proposed approach splits a $K$-class problem into $K-1$ two-class problems. The planning of the one-class-at-a-time removal sequence is formulated as a multistage decision-making problem, which is then solved using dynamic programming.

To reduce the computational cost of the proposed approach, which increases rapidly with the number of classes, a partial decomposition technique is introduced to determine the suboptimal solution. By using the one-class-at-a-time removal sequence to alleviate the ineffective decision problem, this paper also develops a committee machine framework to improve the classification accuracy.

Experimental results show that the proposed approach consistently provides higher classification accuracy than the conventional single classifier 1-a-a and 1-a-1 methods when the number of classes is less than ten. However, for a tested ten-class problem, the computational cost of the proposed approach is three orders of magnitude larger than that of the single classifier method. By applying the partial decomposition technique, this computational cost can be reduced by a factor of about 30 or better. In addition, with the assistance of the proposed committee machine framework, the suboptimal solution produced by the partial decomposition technique provides better classification accuracy than conventional methods.

\section{REFERENCES}

[1] T. G. Dietterich and G. Bakiri, "Solving multiclass learning problems via error-correcting output codes," J. Artif. Intell. Res., vol. 2, pp. 263-286, 1995.

[2] V. Guruswami and A. Sahal, "Multiclass learning, boosting and errorcorrecting codes," in Proc. 12th Annu. Conf. Comput. Learning Theory, 1999, pp. $145-155$.

[3] J. A. Nossek, R. Eigenmann, G. Papoutsis, and W. Utschick, "Classification systems based on neural networks," in Proc. 5th IEEE Int. Workshop Cellular Neural Netw. and Their Applications, 1998, pp. 26-33.

[4] R. Anand, K. Mehrotra, C. K. Mohan, and S. Ranka, "Efficient classification for multiclass problems using modular neural networks," IEEE Trans. Neural Netw., vol. 6, no. 1, pp. 117-124, Jan. 1995. 
[5] S. Knerr, L. Personnaz, and G. Dreyfus, "Single-layer learning revisited: A stepwise procedure for building and training a neural network," in Neurocomputing: Algorithms, Architectures and Applications, ser. NATO ASI Series, F. F. Soulié and J. Hérault, Eds. New York: Springer-Verlag, 1990, vol. F68, pp. 41-50.

[6] C.-W. Hsu and C.-J. Lin, "A comparison of methods for multiclass support vector machines," IEEE Trans. Neural Netw., vol. 13, no. 2, pp. 415-425, Mar. 2002.

[7] D. E. Kirk, Optimal Control Theory. Englewood Cliffs, NJ: PrenticeHall, 1970

[8] A. Verikas, A. Lipnickas, K. Malmqvist, M. Bacauskiene, and A. Gelzinis, "Soft combination of neural classifiers: A comparative study," Pattern Recognit. Lett., vol. 20, pp. 429-444, Apr. 1999.

[9] L. Kuncheva, J. Bezdek, and R. Duin, "Decision templates for multiple classifier fusion: an experimental comparison," Pattern Recognit., vol. 34, pp. 299-314, 1999.

[10] C. L. Blake and C. J. Merz, Dept. Inf. Comp. Sci., Univ. California at Irvine, UCI Repository of Machine Learning Databases Irvine, CA, 1998 [Online]. Available: http://www.ics.uci.edu/ mlearn/ML-Reppository.html

[11] A. Bortoletti, C. Di Fiore, S. Fanelli, and P. Zellini, "A new class of quasi-Newtonian methods for optimal learning in MLP-networks," IEEE Trans. Neural Netw., vol. 14, no. 2, pp. 263-273, Mar. 2003.

[12] C. Xiang, S. Q. Ding, and T. H. Lee, "Geometrical interpretation and architecture selection of MLP," IEEE Trans. Neural Netw., vol. 16, no. 1, pp. 84-96, Jan. 2005.

[13] D. Erdogmus, O. Fontenla-Romero, J. C. Principe, A. Alonso-Betanzos, and E. Castillo, "Linear-least-squares initialization of multilayer perceptrons through backpropagation of the desired response," IEEE Trans. Neural Netw., vol. 16, no. 2, pp. 325-337, Mar. 2005.

[14] Y. Freund and R. E. Schapire, "A decision-theoretic generalization of on-line learning and an application to boosting," J. Comp. Syst. Sci., vol. 55, no. 1, pp. 119-139, 1997.

[15] H. Schwenk and Y. Bengio, "Boosting neural networks," Neural Comput., vol. 12, no. 8, pp. 1869-1887, 2000.

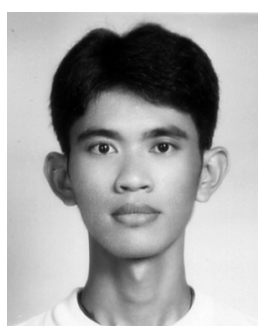

Chieh-Neng Young received the B.E. and M.E. degrees in mechanical engineering from National Sun-Yat University, in 1999 and 2001, respectively, where he is currently working toward the Ph.D. degree.

His research interests include learning in artificial neural networks and pattern recognition.

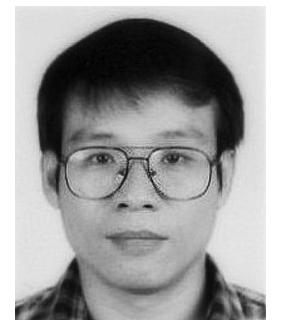

Chen-Wen Yen received the B.E. degree in mechanical engineering from Tamkang University, in 1982, and the M.E. and Ph.D. degrees in mechanical engineering from Carnegie-Mellon University, Pittsburgh, PA, in 1986 and 1989, respectively.

Following graduation, he joined the Faculty of the Department of Mechanical Engineering at Sun Yat-Sen University. His research work focuses on the application of neural networks to pattern recognition problems.

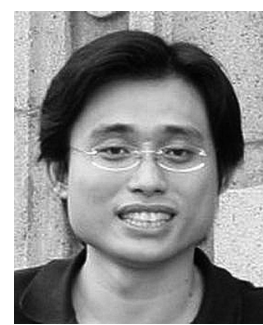

Yi-Hua Pao received the B.E. degree in mechanical engineering from Chung Yuan Christian University, in 2001, and an M.E. degree in mechanical engineering from National Sun-Yat University, in 2003.

$\mathrm{He}$ is currently employed as a Control Systems Engineer at ASUS International (Taiwan).

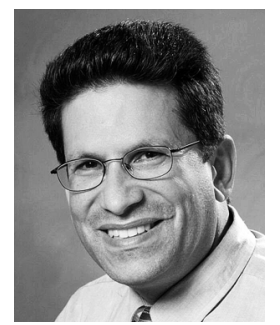

Mark L. Nagurka received the B.S. and M.S degrees in mechanical engineering and applied mechanics from the University of Pennsylvania, Philadelphia, and the Ph.D. degree in mechanical engineering from Massachusetts Institute of Technology, Cambridge.

He joined the Department of Mechanical and Industrial Engineering at Marquette University, Milwaukee, WI after teaching at Carnegie Mellon University, Pittsburgh, PA, and working as a Senior Research engineer at the Carnegie Mellon Research Institute. His research interests include automation, mechatronics, control design, and biomechanics. 